\title{
Determination of the Traffic Properties of Cells with Mobile Users Using a Mixed Traffic
}

\author{
Dragan MITIC, Aleksandar LEBL, Zarko MARKOV, Vladimir KOSJER \\ IRITEL a.d. Beograd, Batajnički put 23, 11080 Belgrade, Serbia \\ \{mita, lebl, zmarkov,vladimir.kosjer\}@iritel.com \\ Submitted June 20, 2019 / Accepted October 18, 2019
}

\begin{abstract}
This paper presents a two-dimensional Markov traffic model of the mobile users' network where there exist handover calls from the surrounding cells to the considered cell and where, also, primary calls are generated. The two emphasized types of calls form together mixed traffic. The new, two-dimensional model allows us to calculate some characteristic variables for the systems, which may not be determined based on the analysis of one-dimensional model. The developed simulation program is verified comparing the obtained system state probabilities as also primary and handover calls loss rate to the corresponding values from the calculation process. We analyzed cells with a number of channels reserved only for handover calls. This system performances are compared to the performances of some other systems from literature and it is proved that their characteristics are comparable whereby our system improves handover calls dropping rate. It is also proved that users' speed increase and cell radius decrease cause both primary and, especially, handover calls loss rate increase. The results of calculation and simulation are obtained after a number of iterations (calculation or simulation cycles), where the new loss probability values from one iteration become the input values for the next iteration. In the case that call loss values do not converge during simulation, we implemented the original algorithm for input call loss probability estimation for the next iteration.
\end{abstract}

\section{Keywords}

Network of mobile users, handover traffic, channel reservation, traffic loss, two-dimensional model

\section{Introduction}

Users' moving in the mobile station (MS) network is enabled by several complex processes. At first, base station (BS) power measurement is applied to select the most suitable one. Then a sophisticated signalization process allows BS changing, i.e. MS transition from one cell to the other, neighboring one (handover). The necessary BS power determination while MSs are moving [1] is also a compli- cated problem, especially when considering power saving. In this paper we are going to apply the theory of mixed (or multi-dimensional, in this case two-dimensional [2]) traffic to present traffic calculation in one mobile telephony network cell when all users are movable. The theory of multidimensional traffic may be implemented when the same serving resources are utilized by users (requests) of different properties. The benefit of this model is that all traffic components properties may be determined. Section 2 presents existing solutions survey, dealing with the analysis of mobile networks with handover traffic component. The adopted cell model with movable users is presented in Sec. 3. Section 4 emphasizes the main steps in the model development, while Section 5 deals with the method of calculation. Section 6 is devoted to the simulation of a cell with handover traffic. Numerical examples are found in Sec. 7. The explanation related to the necessary number of iterations in calculation and simulation process is emphasized in Sec. 8. At the end, the conclusions are expressed in Sec. 9.

\section{Previous Solutions}

Specifics of handover traffic from the point of traffic analysis are established relatively long ago. Basic items related to such an analysis are listed already in [3]: implementation of Markov chains and Erlang loss formulas when defining models, calculation of newly generated calls (primary calls) blocking probability in the considered cell and call dropping probability of handover calls, offered and served traffic of primary and handover calls. The models for various macro and micro mobile network cells configurations are also defined.

The paper [4] is the further elaboration of Markov model presented in [3]. The main expressions for primary calls blocking and handover calls dropping are developed. Besides, the relation between handover intensity, users' moving speed and BS cell dimensions (its perimeter and area) are also introduced. The mentioned expressions are the basis for several examples from praxis presentation in [5]. These examples have been used as a reference point of comparison when developing models presented in our paper. 
The contribution [6] presents detailed analysis of traffic characteristics for the system with handover. The analysis is performed for the three most important methods which aim to achieve call dropping probability decrease: 1) some number of traffic channels reservation only for handover calls; 2) handover calls waiting in a queue for serving and 3) the bit-rate decreasing in already realized connections to handle handover traffic. The number of reserved (or guard) channels may be dynamically varied according to traffic load [7]. Among the three emphasized methods, the most important for the analysis in this paper is channel reservation only for handover calls. The accent in [6] is related to channel reservation for GPRS traffic. However, the analyzed algorithms in [6] do not provide enough multiple call dropping probability decreasing comparing to primary calls blocking probability. The results are presented for the system with relatively small handover probability $\left(P_{\mathrm{h}}=35 \%\right)$, which is not satisfactory when networks with micro-cells are dimensioned. The analysis examples in this paper proved that special attention has to be devoted to the problem of slow convergence of calculated results to their exact values for greater values of handover probability. Such a situation is the characteristic of micro-cells.

The reservation of some traffic channels only for handover leads to primary calls loss increasing. The characteristic behavior of lost calls is that they are repeated. The analysis of systems with repeated calls where primary and handover calls exist is presented in [8].

Contribution [9] presents the detailed analysis of the system where waiting of handover calls is applied if there are no idle traffic channels. There are two queues of different priority in a system depending on the received signal power and the remaining time that a call may be present in the region between two cells. New calls blocking probability and handover calls dropping probability are determined analytically and by simulation. Queueing disciplines are further expanded in [10], where, besides queueing of handover calls, it is supposed also that primary calls are queued.

Newly generated requests arrival to some queueing system may be considered as Poisson process. Strictly speaking, this assumption is not valid for handover traffic, because it is modified by the loss in surrounding cells where the calls are first generated [11]. It is emphasized in the survey of different contributions and analysis, presented in [11], that handover traffic is more smoothed than Poisson process. However, in the case of: 1) small or medium traffic load; 2) even distribution of offered traffic in identical neighboring cells according to Poisson process and 3) relatively limited users' moving dynamics, i.e. when the great number of cells are not exchanged during the connection time, Poisson model represents handover traffic very well [8], [11]. This is very important as the background to approve credibility of the results presented in this paper. The assumptions in the analysis performed in this paper include approximately equal traffic load in cells and limited handovers number during one connection time.
Even in the case when mean users' moving speed is $V=60 \mathrm{~km} / \mathrm{h}$, it is unlikely to exchange more than two cells during the connection, because it is assumed that cell perimeter is $R=10 \mathrm{~km}$. These values of mean users' speed $V$ and cell perimeter $R$ are characteristic for the cells in rural areas. In this paper we analyze such an example. The cells with lower value of $V$ and $R$ are typical for urban areas (especially when microcells are considered). The examples in this paper are for $V=5 \mathrm{~km} / \mathrm{h}$ and $R=100 \mathrm{~m}$. Besides, in this paper are analyzed characteristics of macrocells in rural $(R=10 \mathrm{~km})$ and urban area $(R=1 \mathrm{~km})$ where users' speed is variable. Nevertheless, in the conditions of greater users' moving speed it is necessary to carefully anticipate the moment of new cell selection when handover is realized [12]. Such an analysis decreases the number of unnecessary handovers due to the lack of idle channels or idle waiting spaces, but also it is necessary to implement new, specific traffic models [13]. As, generally, handover traffic is more smoothed than Poisson process, the practically expected results in the case of the significant traffic load and smaller cells are better than the results presented by Poisson model. The analysis of handover traffic is not only important for the improved dimensioning of traffic channels. It may be also implemented to better design the handover algorithm in heterogeneous technology networks [14].

The main contribution of this article is that it introduces two-dimensional model for the analysis of handover traffic. In such models primary traffic and handover traffic are represented separately, as they also appear. This is a step forward comparing to previous solutions based on one-dimensional models where these two traffic components are presented together, as one state [4], [5]. The outputs from the analysis of two-dimensional models are substate probabilities, where each sub-state is defined by two numbers related to each of two traffic components. From these sub-state probabilities it is possible to calculate state probabilities, all types of traffic loss, i.e. any desired characteristic. In the following sections some specific system states (sub-states) are analyzed, which cannot be analyzed by the implementation of one-dimensional models (for example probability that there are no primary calls in the system, probability that there are no handover calls in the system and the probability that there are the same number of primary and handover calls in the system). The analysis on the basis of multi-dimensional traffic models is a very powerful mean for queueing system analysis, as it is illustrated in [15], where the applied two-dimensional traffic model has allowed us to prove that blocking probability, calculated in [16] on the basis of one-dimensional model, is underestimated comparing to the exact value of blocking probability.

The second contribution of the article is the developed special simulation method with iterations, which was not applied earlier. Its implementation is necessary because one traffic component (handover traffic) is not exactly defined in the beginning of simulation, but it depends on the traffic characteristics in the surrounding cells. Starting from some arbitrarily selected value of handover traffic, 
during iterations in simulation this traffic gradually approaches its exact value.

The results from this paper address circuit switched systems, meaning that the results are related to GSM like systems.

\section{Model of Mixed Traffic in Mobile Networks}

Throughout of this paper the following abbreviations are used:

$-\lambda_{\mathrm{p}}-$ intensity of primary calls generation;

$-\lambda_{\mathrm{h}}-$ intensity of handover calls generation;

- $V$ - user moving speed;

$-R$ - cell radius;

$-A_{\mathrm{p}}$ - the offered traffic of primary calls;

- $A_{\mathrm{h}}$ - the offered traffic of handover calls;

$-\mu_{\mathrm{p}}-$ call service intensity of primary calls;

$-\mu_{\mathrm{h}}-$ call service intensity of handover calls;

$-\mu_{\mathrm{c}}-$ channel service intensity;

$-r$ - number of guard channels intended only handover calls;

- $N$ - total number of traffic channels;

$-P_{\mathrm{bp}}$ - probability of primary calls blocking;

$-P_{\mathrm{bh}}-$ probability of handover calls dropping;

$-P_{\mathrm{h}}$ - probability of handover;

$-N_{\mathrm{p}}-$ the number of primary calls in the system;

$-N_{\mathrm{h}}$ - the number of handover calls in the system;

- $P\{i, j\}$ - sub-state probability with $i$ primary calls and $j$ handover calls;

- $P\left(N_{\mathrm{p}}=N_{\mathrm{h}}\right)$ - probability of the substate with $N_{\mathrm{p}}$ primary calls and $N_{\mathrm{h}}$ handover calls;

- sum_P\{0,j\} - probability that no channel in the system is seized by primary call;

- sum_P $\{i, 0\}$ - probability that no channel in the system is seized by handover call;

- $R F(i, N-r-i)$ - the part of handover calls relative frequency which appear in this substate $\{i, N-r-i\}$;

- $P_{\text {bpo }}$ - the old probability of primary calls blocking;

- $P_{\text {bho }}$ - the old probability of handover calls blocking;

- $P_{\mathrm{bpn}}$ - the new probability of primary calls blocking;

- $P_{\text {bhn }}$ - the new probability of handover calls blocking.

The systems with more than one traffic type (usually two types) have been analyzed long ago [17]. Traffic in one cell of mobile network may be considered to have two components. The first one corresponds to calls generated in the considered cell (primary calls). The second one are the calls generated in some other cell, but must be realized, i.e. continued in the considered cell due to users' moving (handover calls). The both traffic types use the same resources, i.e. channels. It is often supposed that handover calls must have service priority. That is to say, call loss due to the resources lack at the connection beginning is considered as common phenomenon. On the contrary, handover connection dropping during the handover is very undesirable. Handover calls priority is achieved by channel

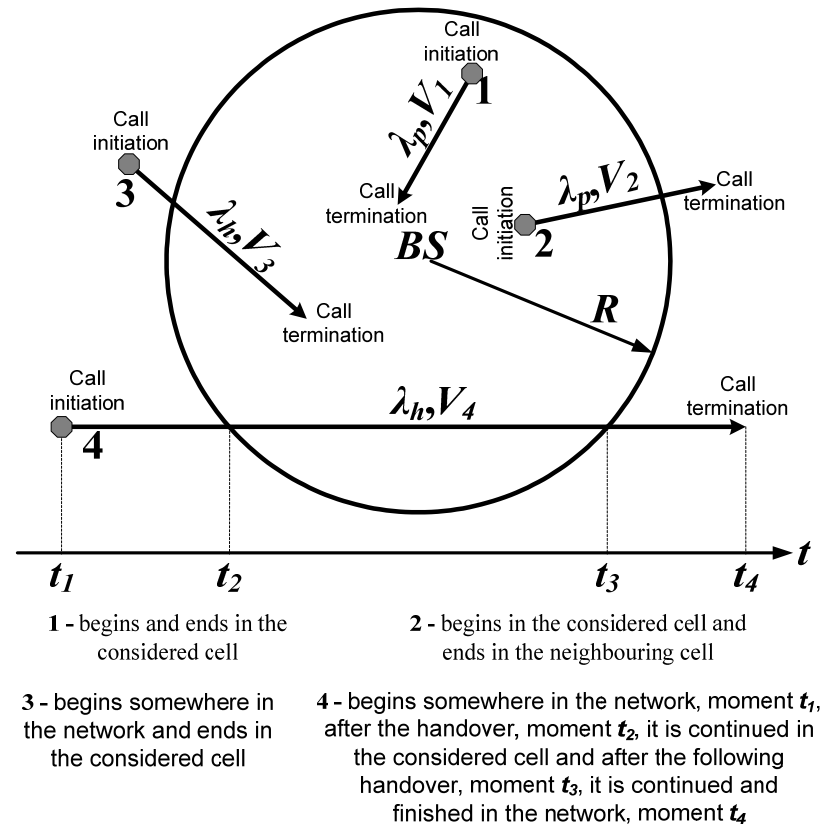

Fig. 1. Symbolic presentation of the cell with movable users.

reservation only for these calls. (In this case handover is realized using two old techniques, which were well known in different stages of classic telephony development. The first one is existing connections rearrangement using other resources [18]. The other one is channel (trunk) reservation [2, Sec. 8.6].)

The circle in Fig. 1 symbolically presents one cell of a mobile network and possible connections while considering the connection beginning and end. The base station (BS) is in the middle of the cell, whose radius is $R$. The number 1 designates the movable user's connection, which begins and ends in the considered cell. Handover is not necessary to be implemented during this connection. The number 2 designates the connection, which starts in the considered cell, and after the realized handover, it continues in the neighboring cell. The connection 3 starts somewhere in the network and after the handover it continues in the considered cell, where it also finishes.

The connection 4 is initiated in the network in the time moment $t_{1}$, after the handover in the moment $t_{2}$ it is continued in the considered cell and after the following handover in the moment $t_{3}$ it is further continued in the network. This connection is finished in the network in the moment $t_{4}$. The time moments on $t$ axis are related only to the user number 4 . It is obvious that, from the point of calls serving, connections 1 and 2 represent the first traffic type for the considered cell (newly generated requests), while the connections 3 and 4 represent the second traffic type (handover calls). The users moving speeds are different $\left(V_{1}-V_{4}\right)$ and the directions of their moving are random.

Let us consider the cell of mobile network with movable users. The cell is circular and its radius is $R$, Fig. 1 . It is supposed that users are uniformly distributed over the cell area. The mean user moving speed is designated by $V$, as presented in Fig. 1. The network consists of the cells 
with equal properties. Each cell has $N$ traffic channels. The calls are generated randomly and do not depend on channel availability state, which means that the number of traffic sources is significantly greater than the number of traffic channels. This assumption is valid both for primary traffic and for handover traffic, because the handover traffic into some cell is the consequence of previously generated primary traffic of users, whose number is always significantly greater than the number of traffic channels. According to Fig. 1, primary calls are generated in the cell with the intensity $\lambda_{\mathrm{p}}$, (calls in the unit of time) and the incoming calls intensity into the cell by handover realization is $\lambda_{\mathrm{h}}$. The offered traffic will be designated by $A$ and will present the product of the call intensity and mean connection time $\left(A_{\mathrm{p}}\right.$ in the case of primary calls the offered traffic, and $A_{\mathrm{h}}$ in the case of handover calls the offered traffic). The time interval between successive calls of both kinds is considered to be random variable with negative exponential distribution. These two traffic components have Poisson distribution, according to [9], [11]. The important assumption is the balance of handover process: the number of calls which come to the considered cell as the result of handover is equal to the number of calls which go from the same cell to other cells also by handover. The mean time while the movable user is in the cell (dwell time) is equal $1 / \mu_{\mathrm{h}}$. As it is known [4], the intensity of handover in the circular cell is $\mu_{\mathrm{h}}=2 \cdot V / \pi \cdot R$. The second consequence of the balance between outgoing and incoming handover connections is that handover probability for one connection is $P_{\mathrm{h}}=\mu_{\mathrm{h}} /\left(\mu_{\mathrm{h}}+\mu_{\mathrm{p}}\right)$. The connection duration time is random variable with the negative-exponential distribution whose mean value is $t_{\mathrm{m}}=1 / \mu_{\mathrm{p}}$, meaning that $\mu_{\mathrm{p}}$ is call service intensity (service rate). The mean channel serving time in one cell is random variable with the negative-exponential distribution, whose mean value is $t_{\mathrm{c}}=1 /\left(\mu_{\mathrm{h}}+\mu_{\mathrm{p}}\right)=1 / \mu_{\mathrm{c}}$. If users are motionless, then it is $t_{\mathrm{m}}=t_{\mathrm{c}}$ and if they move in the network, then it is $t_{\mathrm{m}}>t_{\mathrm{c}}$. If some number of channels is reserved only for handover calls, their number is designated by $r(0 \leq r<N)$. The probability of primary calls blocking due to the lack of idle channels is designated by $P_{\mathrm{bp}}$ and the probability of handover calls dropping is designated by $P_{\mathrm{bh}}$. If it is $r=0$ then it is $P_{\mathrm{bp}}=P_{\mathrm{bh}}$. If in some moment of time $i$ channels in the cell are seized by primary calls and $j$ channels are seized after handover, it will be considered that the cell is in the sub-state $\{i, j\}$ and the probability of this sub-state will be designated $P\{i, j\}$.

The service in a cell may be explained by the simplified model in Fig. 2. The circle in this figure presents the cell with the BS in its middle. The primary calls are generated by the users from the considered cell with the intensity $\lambda_{\mathrm{p}}$ and they are served if there are less than $N-r$ busy traffic channels. If the number of busy traffic channels is $N-r$ or higher, the primary call is blocked. The intensity of blocked calls is $\lambda_{\mathrm{p}} \cdot P_{\mathrm{bp}}$.

Handover calls are generated with the intensity $\lambda_{\mathrm{h}}$ when users from adjacent cells are crossing the cell border. The call is served if some of total $N$ traffic channels is idle. If all $N$ traffic channels are busy, the call is dropped. The

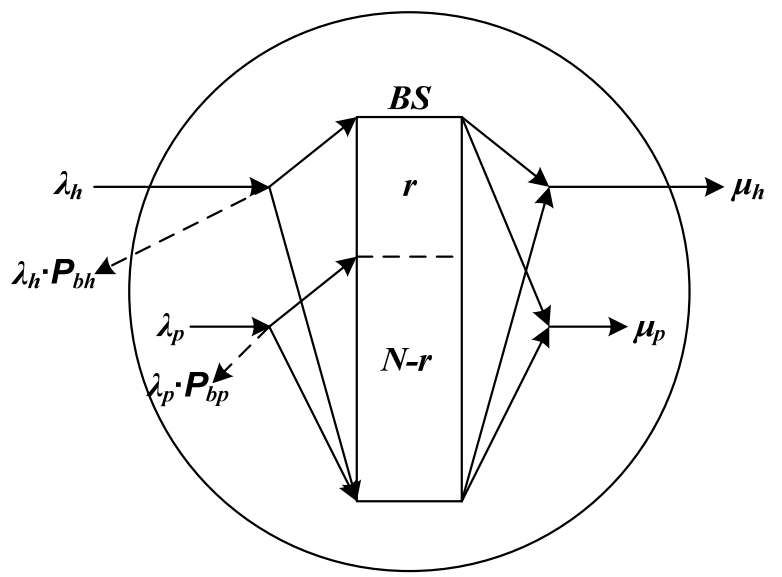

Fig. 2. Serving of primary and handover calls in a cell.

intensity of dropped handover calls is $\lambda_{\mathrm{h}} \cdot P_{\mathrm{bh}}$. There is no queueing possibility in the system for primary calls or for handover calls.

All calls accepted for service may be ended while a user is still in the considered cell. The intensity of these calls finishing is $\mu_{\mathrm{p}}$. The call service in the considered cell may be finished before the call ends by handover to some of adjacent cells. The intensity of these calls is $\mu_{\mathrm{h}}$.

\section{The Analysis Steps}

Traffic process takes place under the influence of two traffic components. That's why this process may be presented as two-dimensional [2, Sec. 7]. In the case of reserved channels implementation $(r>0)$ the process comes to the case of one traffic type restriction [2, Sec. 8.6.1.]. This case is presented by the model in Fig. 3 where $N=5$, $r=2$. In this figure index $i$ represents the number of primary calls in the system and index $j$ represents the number of handover calls. The number of channels reserved for handover calls is related to the maximum possible values of $i$ and $j$ by $r=j-i$. Sub-state diagram, i.e. all possible sub-states in such a cell and transition possibilities from one sub-state to the other are presented in Fig. 4. Each substate in Fig. 4 is described by two indices. The first index is the instantaneous number of primary calls in the system and the second index is the number of handover calls in the system. If Kolmogorov criterion [2] is implemented on the transition diagram from Fig. 4, it is proved that the process in this model is not reversible. That's why the simpler calculation method may not be implemented.

The necessary condition of two-dimensional process reversibility according to Kolmogorov criterion [2] is that process flow between neighboring sub-states must exist in both directions and this is not the case for the system with channel reservation in Fig. 4. That's why the calculation of sub-states probabilities is more complex than for reversible process. Determination of traffic properties for this model will be realized in three steps. The first step is accurate properties calculation for the model with the small number of sub-states. This is relatively simple. The second step is 


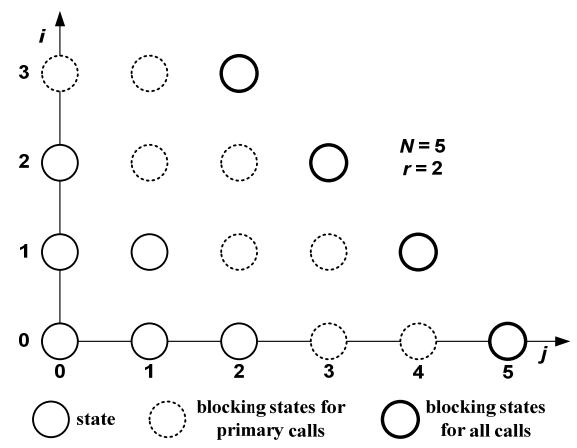

Fig. 3. Sub-states in two-dimensional traffic model of one cell.

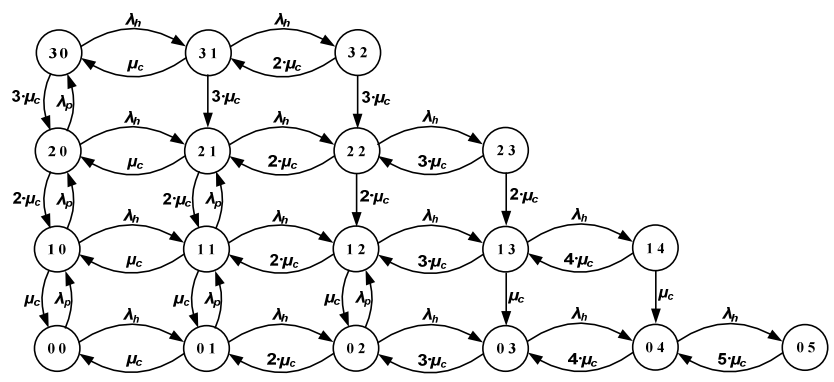

Fig. 4. Sub-state diagram for the cell from Fig. 3.

development of simulation model for the calculated model. The third step is implementation of simulation on a model with greater number of sub-states.

\section{Calculation Method}

Queueing systems like the one in Fig. 4 may often be encountered when analyzing in different areas of science, including situations of everyday life. One such example can be found in [19], wherein it is described how we can come first to a system of differential equations that describe such a case on the basis of the sub-state diagram. In the next step the system of differential equations is replaced in the steady state by a system of linear equations.

In our analysis this system of linear equations is solved by matrix calculation in Excel for systems with a relatively small number of channels $N$, as in [19]. The sub-state probabilities $P\{i, j\}, i=0,1, \ldots, N-r ; \mathrm{j}=0,1, \ldots, N$ are obtained as the result of calculation. These probabilities allow us to determine all traffic parameters for both traffic types.

The probability of both traffic types loss (primary calls blocking and handover calls dropping) is

$$
P_{\mathrm{t}}=\sum_{i=0}^{i=N-r} P\{i, N-i\} \text {. }
$$

The variable $P_{\mathrm{t}}$ in (1) represents the probability of the system state where all $N$ traffic channels are busy. This probability is calculated as the sum of probabilities of twodimensional system sub-states. In Sec. 3 we have already emphasized that the number of traffic sources is signifi- cantly greater than the number of available traffic channels. According to the queueing theory analysis [2] for systems with Erlang property (great number of traffic sources), three values have equal values: 1 . the state probability where all serving channels are busy; 2 . the probability of new calls blocking (call congestion) and 3. the probability of blocking in time (time congestion). This consideration allowed us to implement the equation (1).

The probability of only primary calls blocking is

$$
P_{\mathrm{pl}}=\sum_{i=0}^{N-r} \sum_{j=N-r}^{N-1} P\{i, j-i\} .
$$

The total primary calls blocking rate is

$$
P_{\mathrm{bp}}=P_{\mathrm{t}}+P_{\mathrm{pl}} \text {. }
$$

The handover calls dropping rate is

$$
P_{\mathrm{bh}}=P_{\mathrm{t}} \text {. }
$$

The sub-state probabilities may be used to calculate other variables. For example, system is in the state with $s$ busy channels if it is $i+j=s$, i.e. probability of this state is

$$
P\{s\}=\sum_{i=0}^{i=\min (s, N-r)} P\{i, s-i\}, s=0,1, \ldots, N
$$

where $i=\min (s, N-r)$ means that the upper sum limit is the lower of two offered values: $s$ or $N-r$.

The probability that a cell is in the state with $q$ busy channels by primary calls is

$$
P_{\mathrm{p}}\{q\}=\sum_{j=0}^{N-q} P\{q, j\}, q=0,1, \ldots, N-r .
$$

The probability that a cell is in the state with $v$ busy channels by handover calls is

$$
P_{\mathrm{h}}\{v\}=\sum_{i=0}^{i=\min (N-v, N-r)} P\{i, v\}, v=0,1, \ldots, N
$$

where $i=\min (N-v, N-r)$ means that the upper sum limit is the lower of two offered values: $N-v$ or $N-r$.

Other traffic characteristics (offered traffic in substates) may be determined on the basis of sub-state probabilities.

Unfortunately, sub-state probabilities may not be determined in one process flow. Iterative process must be used [5]. The reason for this is simple: the offered traffic to channels in the considered cell depends on the offered handover traffic and this handover traffic depends on loss in neighboring cells.

The offered traffic to the considered cell from the neighboring cells, Fig. 5, depends on handover probability $P_{\mathrm{h}}$. Handover is used to transmit only served primary calls $\left(1-P_{\mathrm{bp}}\right) \cdot A_{\mathrm{p}}$ from the neighboring cells and served calls, which are present in neighboring cells as a consequence of 


\section{All the neighboring cells}

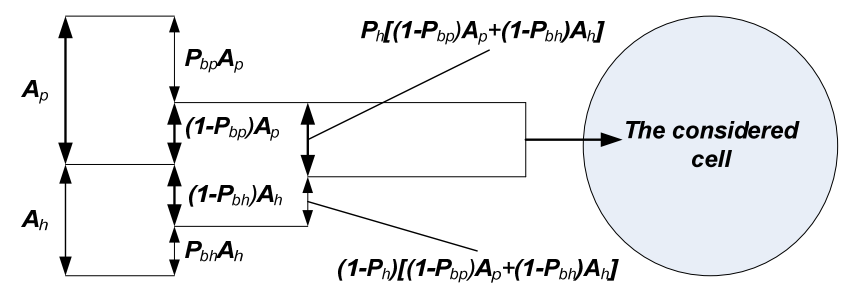

Fig. 5. Traffic process in the considered cell on the basis of handover.

handover $\left(1-P_{\mathrm{bh}}\right) \cdot A_{\mathrm{h}}$. The value of $A_{\mathrm{h}}$ is determined on the basis of equation

$$
A_{\mathrm{h}}=P_{\mathrm{h}} \cdot\left[\left(1-P_{\mathrm{bp}}\right) \cdot A_{\mathrm{p}}+\left(1-P_{\mathrm{bh}}\right) \cdot A_{\mathrm{h}}\right]
$$

i.e.

$$
A_{\mathrm{h}}=\frac{P_{\mathrm{h}} \cdot\left(1-P_{\mathrm{bp}}\right) \cdot A_{\mathrm{p}}}{1-\left(1-P_{\mathrm{bh}}\right) \cdot P_{\mathrm{h}}} .
$$

The offered traffic of primary calls is a priori defined for sub-states probabilities calculation, but the offered handover traffic depends on $P_{\mathrm{bp}}$ and $P_{\mathrm{bh}}$ loss. The values of $P_{\mathrm{bp}}$ and $P_{\mathrm{bh}}$ are assumed at the beginning of calculation. The calculation process is realized in several iterations of sub-state probabilities and loss calculation. The new values of loss (primary calls blocking and handover calls dropping) are used in (9). The new values of $P_{\mathrm{bp}}$ and $P_{\mathrm{bh}}$ are used for the next iteration steps until the value of $P_{\mathrm{bp}}$ at the end of calculation is close enough to the value before the calculation. In practical realization we have finished the calculation when the value of $P_{\mathrm{bp}}$ at the end of some iteration does not differ more than $d i f=1 \%$ or $d i f=1.5 \%$ from its value before the calculation.

\section{Simulation Method}

The process of simulation is based on classical Monte Carlo simulation of telephone traffic, which is explained, for example, in [20]. This method is modified in accordance to the requirements of specific system which is analyzed, whether it is a system in telephony [21], or in some other area [22]. The procedure of characteristic events generation is adjusted considering the specificity of analyzed system, as is usually also the case in other examples of Markov traffic (process) simulation.

The main characteristic of Markov traffic (process) is that previous events have no influence on following events (memoryless property). This characteristic is provided by the generator of random numbers without the influence of past events. Each generated random number causes maximum one change (the new call beginning or finishing). In this way it is assured that simulated process has the birthdeath property as the real process.

The important difference in simulation flow comparing to some other earlier realized simulations [21-23] is that the final simulation result is obtained after more simulation cycles (iterations), not after only one. This specificity is the consequence of the fact that handover traffic may not be uniquely defined. Its value depends on $P_{\mathrm{bp}}$ and $P_{\mathrm{bh}}$ values in the adjacent cells and after each cycle the new value of $A_{\mathrm{h}}$ is calculated for the next cycle on the basis of $P_{\mathrm{bp}}$ and $P_{\mathrm{bh}}$ determined in the just finished cycle. This is the principle difference comparing to simulations in [21-23]. There are also other differences in the simulation flow connected with the process of events generation, which are not principle. It means that simulation programs from [21-23] couldn't be directly modified to perform more iterations, but it was necessary also to redefine events generation behavior and the areas of generated random numbers to correspond to the system modelled in this paper.

Each simulation cycle in our program includes relatively great number of generated random numbers (and events in the simulated system on the basis of these numbers), i.e. repeating the program loop. When selecting the number of program loop repetitions, it is necessary to have as higher as possible number of repetitions to achieve as reliable as possible results. But the high number of these repetitions contributes to too long simulation process. In our case each simulation cycle included 25 million of generated random numbers, which is a good compromise from the viewpoint of the results reliability and simulation time.

The whole presented system simulation is our development completely realized in $\mathrm{C}$ programming language, including also Markov traffic model implementation. It is performed on commercial PC without any special requirements for the PC. The simulation program is available at the address http://www.iritel.com/index.php/en/designservices/646-pprgrams-for-mobile-systems-simulation [24]. We do not use any already existing program for the Markov chain simulation. Our program as all other programs which we have developed for the simulations of mobile systems function we treat as the open source software and we disclose it to anyone who is interested. The time needed for each simulation trial with 25 million of generated random numbers is less than $10 \mathrm{~s}$. Simulation starts by arbitrary defining the starting values of traffic loss rate $P_{\mathrm{bp}}$ and $P_{\mathrm{bh}}$ (for example $P_{\mathrm{bpn}}=P_{\mathrm{bhn}}=0$ ) (Fig. 6). After that the first step in the simulation is the generation of random number $R N$ with the uniform distribution in the range $(0, \ldots, 1)$ (classical random number generator in $\mathrm{C}$ programming packet). This number is, afterwards, translated to the range $\left(0, A_{\mathrm{p}} \cdot(1+\Delta)+N\right)$, where $\Delta=A_{\mathrm{h}} / A_{\mathrm{p}}$ defines the part of offered traffic which is the subject of handover. The handover traffic is calculated on the basis of (9). Depending on the calculated random number $R N 1$ the following events are possible:

- $R N 1<A_{\mathrm{p}}-$ the primary call is generated if the number of busy channels in that moment is $N_{\mathrm{t}}<N-r$; if this condition for $N_{\mathrm{t}}$ is not satisfied, the primary call is lost $\left(L_{\mathrm{p}}\right)$; 
- $A_{\mathrm{p}} \leq R N 1<A_{\mathrm{p}} \cdot(1+\Delta)-$ the handover call is generated if the number of busy channels in that moment is $N_{\mathrm{t}}<N$; if this condition for $N_{\mathrm{t}}$ is not satisfied, the handover call is lost $\left(L_{\mathrm{h}}\right)$;

- $A_{\mathrm{p}} \cdot(1+\Delta) \leq R N 1<A_{\mathrm{p}} \cdot(1+\Delta)+N_{\mathrm{p}}$ - the primary call is finished if the number of existing primary calls in the channels in that moment is $N_{\mathrm{p}}>0$;

- $A_{\mathrm{p}} \cdot(1+\Delta)+N_{\mathrm{p}} \leq R N 1<A_{\mathrm{p}} \cdot(1+\Delta)+N_{\mathrm{p}}+N_{\mathrm{h}}-$ the handover call is finished if the number of existing handover calls in the channels in that moment is $N_{\mathrm{h}}>0$;

- $R N 1 \geq A_{\mathrm{p}} \cdot(1+\Delta)+N_{\mathrm{p}}+N_{\mathrm{h}}-$ no events are generated.

During the simulation the record is kept about the number of generated and lost primary and handover calls. At the end of simulation cycle when the instantaneous value of program loop passes counter (cnt) approaches its maximum value $(\max )$, we calculate from these data new probabilities $P_{\mathrm{bpn}}$ and $P_{\mathrm{bhn}}$. The new value $P_{\mathrm{bpn}}$ is compared to the old value of the probability $P_{\mathrm{bpo}}$. The described procedure is repeated until relative difference between the old (at the input to the instantaneously realized iteration) and the new (after the realized iteration) value of $P_{\mathrm{bp}}$ does not become lower than dif. During this procedure equation (9) is used to calculate the new value of $A_{\mathrm{h}}$, i.e. $\Delta$ on the basis of the new values of $P_{\mathrm{bpn}}$ and $P_{\mathrm{bhn}}$.

\section{Numerical Examples}

Accuracy of simulation model for the analysis of systems with handover is verified for the cell modelled by Fig. 3 with the corresponding sub-state diagram from Fig. 4. For such a cell Figure 7 gives sub-states probabilities obtained by calculation and the mean values obtained on the basis of simulation after at least three simulation trials, for the offered traffic value $A_{\mathrm{p}}=2 \mathrm{E}$. In our terminology each simulation trial consists of a number of simulation cycles (iterations) until the value $P_{\mathrm{bp}}$ changes less than dif in two consecutive cycles. In our simulation each performed cycle consists of several million passes through the program loop. The BS cell radius is $R=10 \mathrm{~km}$, and the mean users' velocity is $V=60 \mathrm{~km} / \mathrm{h}$, leading to the handover probability $P_{\mathrm{h}}=0.17$ [5]. The values of $P_{\mathrm{bp}}$ and $P_{\mathrm{bh}}$ are presented at the end of Fig. 7. The values of $P\{i, j\}, P_{\mathrm{bp}}$ and $P_{\text {bh }}$ are selected in the moment when the relative difference between the values of $P_{\mathrm{bp}}$ after two consecutive, last iterations is lower than $1 \%$.

The next step in the verification was the comparison of probability loss values $P_{\mathrm{bp}}$ and $P_{\mathrm{bh}}$, according to the calculation and the simulation with the values on the basis of one-dimensional model, presented in [5]. The results of this verification are emphasized in Tab. 1 .

The comparison is performed for two examples: the first one where $r=2$ channels are reserved only for handover and the second one where no channel is reserved for handover $(r=0)$. In the case of $r=0$ the values of $P_{\mathrm{bp}}$ and

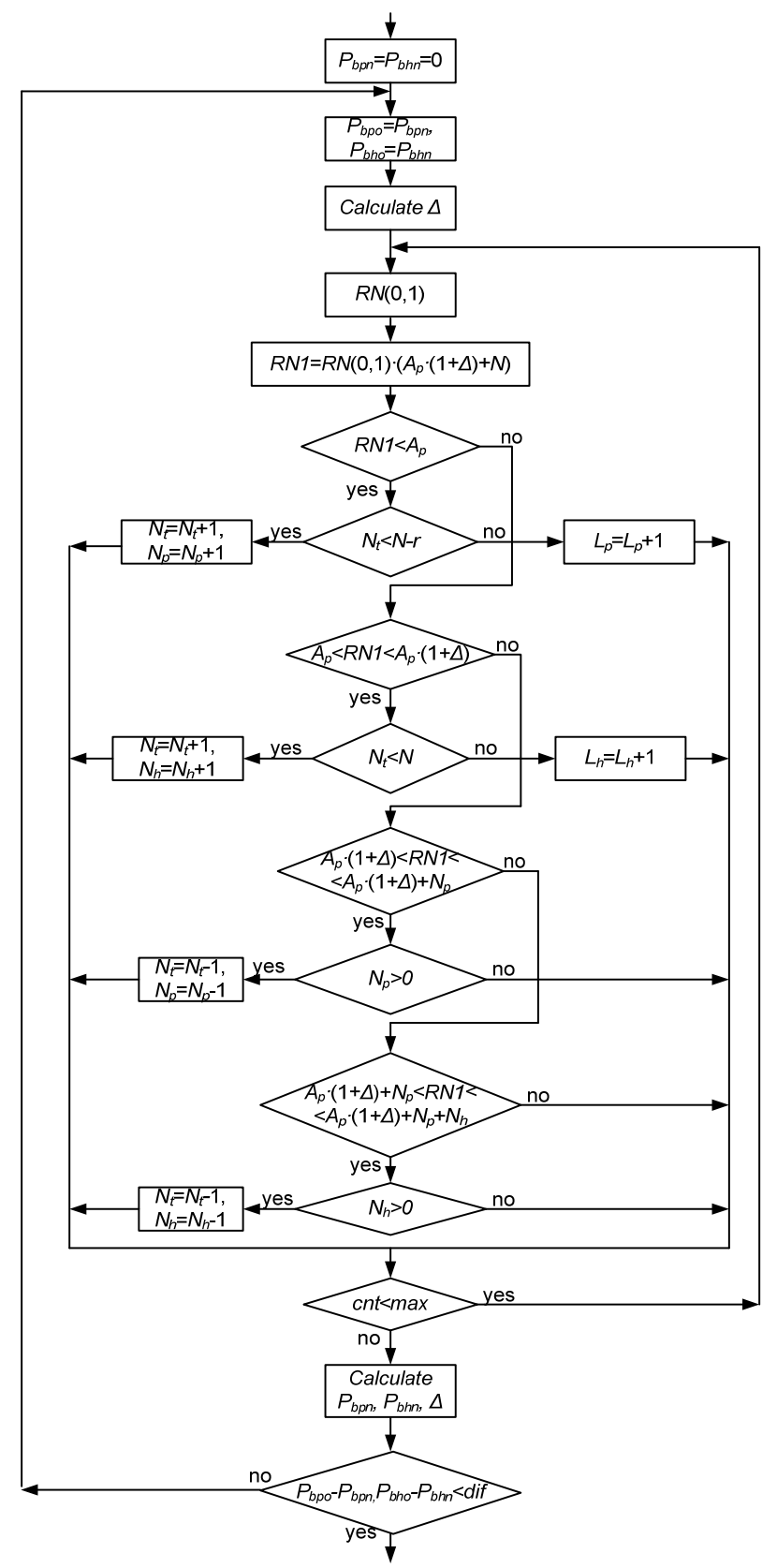

Fig. 6. Flow-chart of the simulation program.

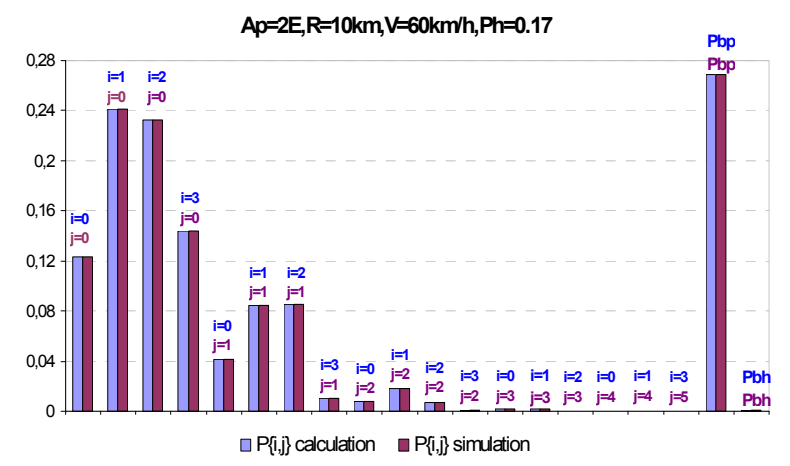

Fig. 7. The sub-state probabilities $P\{i, j\}$ and the probability of primary calls blocking $\left(P_{\mathrm{bp}}\right)$ and handover calls dropping $\left(P_{\mathrm{bh}}\right)$ on the basis of calculation and the mean value of the same variables on the basis of simulation for the system presented in Fig 3 and Fig. 4. 


\begin{tabular}{|c|c|c|c|}
\hline & One-dimensional model in [5] & Calculation & Mean value of simulation \\
\hline$P_{\mathrm{bp}}$ for $N=10, r=2, A_{\mathrm{p}}=5 \mathrm{E}, P_{\mathrm{h}}=0.17$ & 0.12698 & 0.126975679 & 0.127079 \\
\hline$P_{\mathrm{bh}}$ for $N=10, r=2, A_{\mathrm{p}}=5 \mathrm{E}, P_{\mathrm{h}}=0.17$ & 0.0010172 & 0.001017208 & 0.001003 \\
\hline$P_{\mathrm{bp}}$ for $N=10, r=0, A_{\mathrm{p}}=5 \mathrm{E}, P_{\mathrm{h}}=0.17$ & 0.042299 & 0.042300886 & 0.042309 \\
\hline$P_{\mathrm{bh}}$ for $N=10, r=0, A_{\mathrm{p}}=5 \mathrm{E}, P_{\mathrm{h}}=0.17$ & 0.042299 & 0.042300886 & 0.042630 \\
\hline
\end{tabular}

Tab. 1. The probabilities of primary calls blocking $\left(P_{\mathrm{bp}}\right)$ and handover calls dropping $\left(P_{\mathrm{bh}}\right)$ for $N=10, A_{\mathrm{p}}=5 \mathrm{E}, R=10 \mathrm{~km}, V=60 \mathrm{~km} / \mathrm{h}$ and $P_{\mathrm{h}}=0.17$ in the case when it is $r=0$ and $r=2$, which are determined on the basis of one-dimensional model according to [5], and on the basis of calculation and simulation for the new two-dimensional model.

$P_{\text {bh }}$ obtained by calculation are exactly the same. This is understandable because $P_{\mathrm{bp}}$ and $P_{\mathrm{bh}}$ are determined as the sum of system sub-states probabilities, and when it is $r=0$ there are no sub-states where only primary calls are lost. When simulation is realized, the values of $P_{\mathrm{bp}}$ and $P_{\mathrm{bh}}$ are very near one to the other, but not the same. The reason is that the loss in simulation is determined in a different way: by counting lost and successfully realized primary and handover calls. At the end of the simulation, $P_{\mathrm{bp}}$ and $P_{\mathrm{bh}}$ are calculated dividing the numbers of lost and established calls.

Figure 8 presents probability of primary calls blocking $\left(P_{\mathrm{bp}}\right)$ and handover calls dropping $\left(P_{\mathrm{bh}}\right)$ as a function of offered traffic $\left(A_{\mathrm{p}}\right)$ in two cases: 1$)$ when $N=10$ traffic channels are available and $r=2$ channels are reserved only for handover calls; 2) when $N=22$ traffic channels are available and $r=3$ channels are reserved only for handover calls. The handover probability is $P_{\mathrm{h}}=0.17$. The results are determined by simulation.

When there is a higher number of available traffic channels, the number of equations which model the state of the system is significantly increased over the maximum possibilities of the implemented software. That's why in such a case the results may be only determined by simulation (the example of $N=22$ channels in Fig. 8). It may be concluded that reservation of only a low number of channels only for handover calls ( 3 of total 22 channels) causes decreasing of handover calls loss probability in relation to primary calls loss for approximately three orders of magnitude. Such a significant improvement is the result of two mutually opposite effects. The first one is that reservation of $r$ channels only for handover calls causes that the system behaves in relation to primary calls as it has $r$ channels less available. That's why probability of primary calls blocking is increased comparing to the case when there is no channel reservation. The second effect is that only handover calls contribute to the offered traffic on $r$ reserved channels. This is, in any case, lower traffic then if there is no channel reservation, when the offered traffic on these $r$ channels includes, together, primary and handover calls. Therefore, this contributes to handover calls dropping rate decrease.

Comparing the results for the smaller group of 10 channels from Fig. 8 in relation to the effects for the greater group of 22 channels, it may be concluded that the achieved effects of loss decrease are greater for the greater group of channels. For example, for $P_{\mathrm{bp}}=0.1$ in the system

\section{$\mathrm{R}=10 \mathrm{~km}, \mathrm{~V}=60 \mathrm{~km} / \mathrm{h}, \mathrm{Ph}=0.17$}

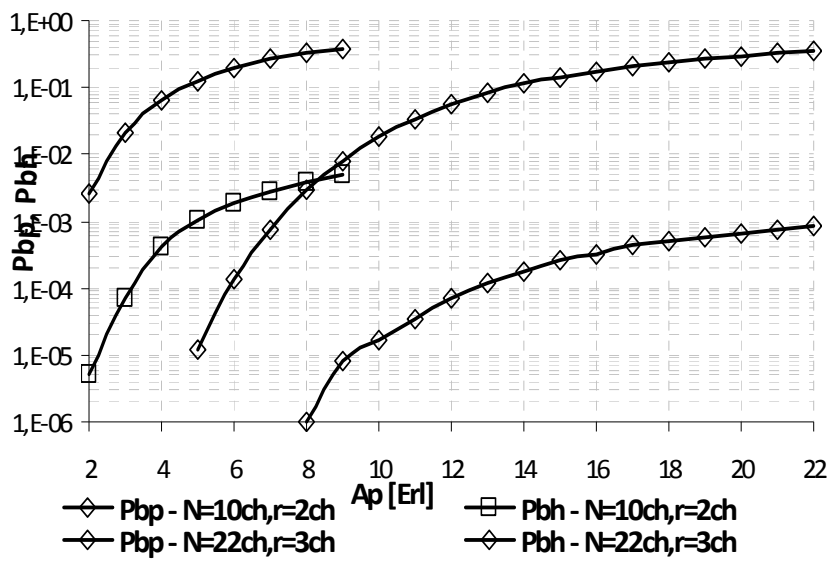

Fig. 8. The probability of primary calls blocking $\left(P_{\mathrm{bp}}\right)$ and handover calls dropping $\left(P_{\mathrm{bh}}\right)$ as a function of offered traffic $\left(A_{\mathrm{p}}\right)$.

with 10 channels it is $P_{\mathrm{bh}} \approx 8 \cdot 10^{-4}$, while for the same value of $P_{\mathrm{bp}}$ in the system with 22 channels is $P_{\mathrm{bh}}<2 \cdot 10^{-4}$. This is achieved although, relatively, the number of reserved channels in relation to the total number of channels is greater for the system with 10 channels $(2 / 10$ in relation to $3 / 22)$.

The advantage of two-dimensional model implementation for the system with handover calls is that it allows us to perceive some system characteristics, which may not be estimated on the basis of one-dimensional model. One example is the probability that there are equal number of realized primary and handover calls $\left(P\left(N_{\mathrm{p}}=N_{\mathrm{h}}\right)\right)$ in the system as a function of offered traffic $\left(A_{\mathrm{p}}\right)$, calculated according to the equation

$$
P\left\{N_{\mathrm{p}}=N_{\mathrm{h}}\right\}=\sum_{i=0}^{\left\lfloor\frac{N}{2}\right\rfloor} P\{i, i\}
$$

where $\left\lfloor\frac{N}{2}\right\rfloor$ is the lower integer value of the number $N / 2$.

For the system defined by Fig. 8 this probability is presented in Fig. 9. When it is $N=10$ the value $\left\lfloor\frac{N}{2}\right\rfloor$ goes from 0 to 5 while when it is $N=22$ it goes from 0 to 11 . It is obvious that probability $P\left\{N_{\mathrm{p}}=N_{\mathrm{h}}\right\}$ decreases when $A_{\mathrm{p}}$ increases. When traffic values are lower $\left(A_{\mathrm{p}}<5 \mathrm{E}\right)$, $P\left\{N_{\mathrm{p}}=N_{\mathrm{h}}\right\}$ is approximately the same for 10 and 22 traffic channels. 
$\mathrm{R}=10 \mathrm{~km}, \mathrm{~V}=60 \mathrm{~km} / \mathrm{h}, \mathrm{Ph}=0.17$

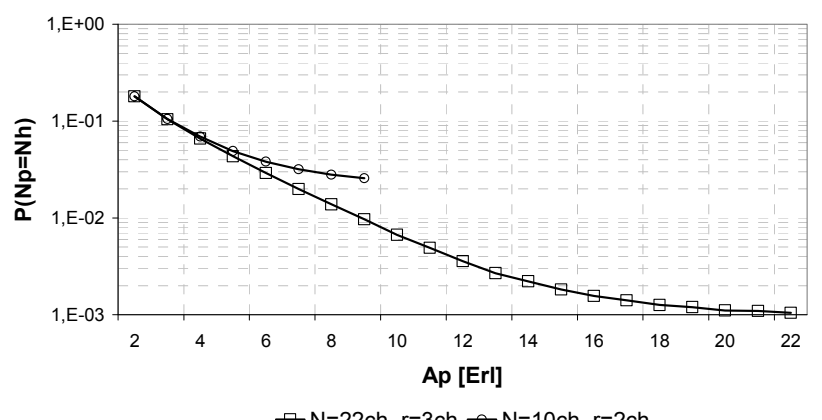

Fig. 9. The total probability that the same number of primary and handover calls is realized as a function of offered traffic $\left(A_{\mathrm{p}}\right)$.

$\mathrm{N}=10 \mathrm{ch}, \mathrm{r}=2 \mathrm{ch}, \mathrm{R}=10 \mathrm{~km}, \mathrm{~V}=60 \mathrm{~km} / \mathrm{h}, \mathrm{Ph}=0.17$

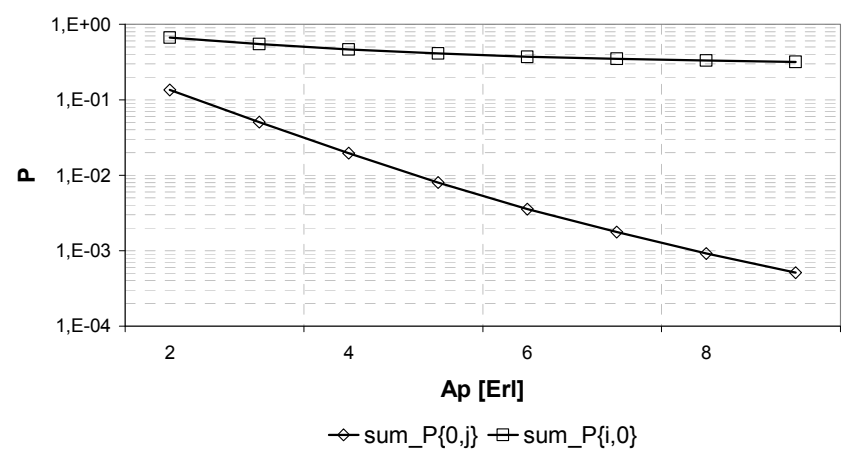

Fig. 10. The probability that in system no channel is seized by primary calls (sum_P\{0,j\}) and that no channel is seized by handover calls (sum_P $P\{i, 0\}$ ) as a function of offered traffic $\left(A_{\mathrm{p}}\right)$.

The probability that in system no traffic channel is seized by primary call (equation (6) for $q=0$ ), i.e. that no traffic channel is seized by handover call (equation (7) for $v=0$ ) also may not be determined by one-dimensional model. In Fig. 10 these probabilities are presented as a function of offered traffic $\left(A_{\mathrm{p}}\right)$ for the cell with $N=10$ channels with $r=2$ channels reserved only for handover calls. It is obvious that these probabilities decrease when $A_{\mathrm{p}}$ increases, significantly faster for the case when there are no primary calls in the system $(q=0)$.

The probabilities of primary and handover calls loss $\left(P_{\mathrm{bp}}\right.$ and $\left.P_{\mathrm{bh}}\right)$ as a function of offered traffic for the microcell with $N=10$ traffic channels are presented in Fig. 11 . The characteristic of these cells is the greater value of handover probability $\left(P_{\mathrm{h}}\right)$ than it is in the case of macrocells, which have been analyzed in this paper until now. For the example from Fig. 11 the cell radius is $R=100 \mathrm{~m}$, the mean users' moving speed is $V=5 \mathrm{~km} / \mathrm{h}$ and $P_{\mathrm{h}}=0.64$ [5]. The value of $P_{\mathrm{h}}$ for microcell is significantly higher than $P_{\mathrm{h}}=0.17$, which is the typical value for the macro-cell used in the analysis in this paper. In regard to the higher value of $P_{\mathrm{h}}$, the greater number of channels is reserved only for handover calls ( $r=4$ in Fig. 11).

The loss probabilities $P_{\mathrm{bp}}$ and $P_{\mathrm{bh}}$ as in Fig. 8 and Fig. 11 may be compared to the corresponding graphs from

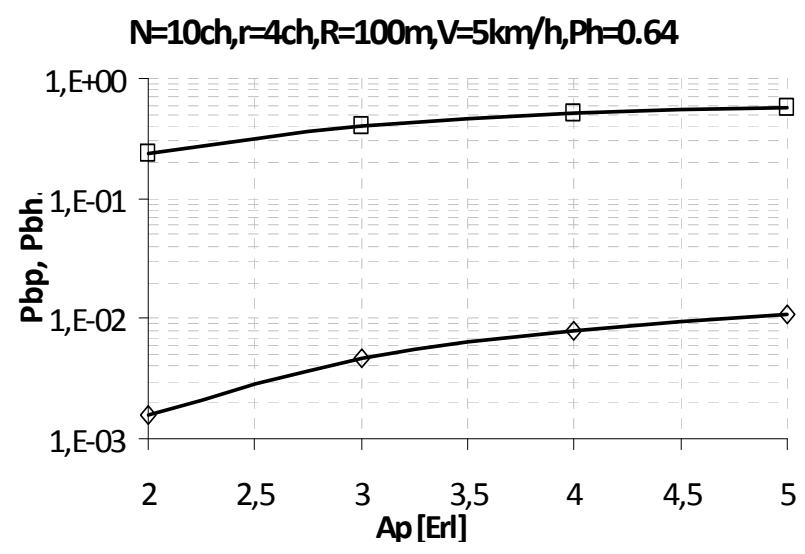

Fig. 11. The loss of primary $\left(P_{\mathrm{bp}}\right)$ and handover calls $\left(P_{\mathrm{bh}}\right)$ as a function of offered traffic $\left(A_{\mathrm{p}}\right)$.

[9] for the system with queueing of handover calls. In order to perform the comparison, we simulated the system with 30 channels where $r=2$ and $r=3$ channels are reserved only for handover calls. It is $P_{\mathrm{h}}=0.5$, as in [9]. The results of simulation according to the cited characteristics together with the results of Fig. 6 from [9] are presented in Fig. 12. The offered traffic in graphic from [9] presents the total traffic of primary calls plus traffic of handover calls. That's why we had to modify our way of presentation to adapt it to the results from [9]. The loss of primary calls for $r=2$ according to our model is higher than the corresponding loss in [9]. When we have $A_{\mathrm{p}}+A_{\mathrm{h}}=20 \mathrm{E}$, it is $P_{\mathrm{bp}}=2.8 \%$ for the system with channel reservation and $P_{\mathrm{bp}}=1.1 \%$ for the system from [9]. For the loss of handover calls the results are opposite: $P_{\mathrm{bh}}=0.21 \%$ for the system with channel reservation and $P_{\mathrm{bh}}=0.25 \%$ for the system from [9]. Relative increase of primary calls loss for system with channel reservation is higher than relative decrease of handover calls loss because calls which may not be serviced are immediately lost. On the other side, handover is faster because no calls are waiting on handover. But, our main goal is to prove the efficiency and the benefits of implemented two-dimensional model, not the benefits of algorithm. As presented in Fig. 12, it is possible to further decrease the probability of handover calls loss by increasing the value of $r(r=3)$, but for a cost of increasing the probability of primary calls loss.

The two systems may be compared in several additional components. The model in [9] does not make difference between arrival of primary and handover calls as long as there are idle traffic channels. In this case system is onedimensional. System is two-dimensional only in the part when there are handover calls, but only because there are two waiting queues. Comparing to this, our model explicitly makes difference between two call types in all situations, thus enabling us to analyze some system characteristics, which are not obvious from one-dimensional model. These are, for example, the probability that system is in some specific sub-state with handover calls present or the probability of a new call generation in different sub-states.

Kolmogorov criterion is satisfied for the system in [9]. This fact allows us to express probabilities of system 


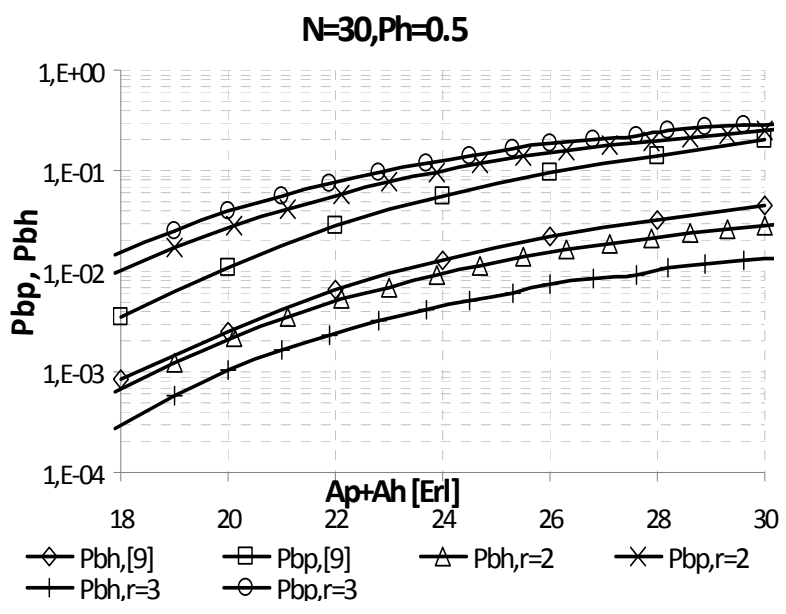

Fig. 12. The probability of primary calls blocking $\left(P_{\mathrm{bp}}\right)$ and handover calls dropping $\left(P_{\mathrm{bh}}\right)$ as a function of total traffic $\left(A_{\mathrm{p}}+A_{\mathrm{h}}\right)$.

states in closed form. Opposite, it is not possible to give such an expression for our system, because some states are not reversible. But, nevertheless, it is possible to calculate the sub-states possibilities solving the system of equations formed on the basis of system birth-death presentation, as the one in Fig. 4.

The characteristics $P_{\mathrm{bp}}$ and $P_{\mathrm{bh}}$ for the model presented in this paper are also compared to the characteristics of the system from [25]. The characteristics from this paper are compared to the characteristics from Fig. 6 and Fig. 7 in [25]. Both systems have 10 traffic channels and we adopted high value $P_{\mathrm{h}}=0.64$ for the system presented in this paper, thus significantly increasing the values of $P_{\mathrm{bp}}$ and $P_{\mathrm{bh}}$. The number of reserved channels only for handover calls is $r=3$. The comparative graphs are presented in Fig. 13. In this case the values of $P_{\mathrm{bh}}$ are mutually similar: for lower values of $A_{\mathrm{p}}<7 \mathrm{E}$ system from [25] has lower $P_{\mathrm{bh}}$, while for $A_{\mathrm{p}}>7 \mathrm{E}$ system from [25] has higher $P_{\mathrm{bh}}$. The value of $P_{\mathrm{bp}}$ is always lower for the system from [25]. But, as already emphasized, the results are presented for the high value $P_{\mathrm{h}}=0.64$, meaning that handovers are very frequent. Besides, as for the comparison with the results from [25], the main goal of this paper is to prove the benefits of two-dimensional model, not to develop the new model for handover implementation.

Performances of a system with handover depend on the cell radius $R$ and on the user speed $V$. These performances are analyzed for the systems with the characteristics presented in Tab. 2. The first three examples are for the systems in rural areas, where $R$ and $V$ are higher, while the second three examples are for the systems in urban areas, where $R$ and $V$ are lower. The values of $P_{\mathrm{h}}$ in the third column of Tab. 2 are determined based on the already emphasized formula from [4]. As the values of $R$ and $V$ affect the value of $P_{\mathrm{h}}$ by their quotient, as an example the values of $P_{\mathrm{bp}}$ and $P_{\mathrm{bh}}$ for the system with $R=10 \mathrm{~km}$ and $V=50 \mathrm{~km} / \mathrm{h}$ are equal to the system with $R=1 \mathrm{~km}$ and $V=5 \mathrm{~km} / \mathrm{h}$.

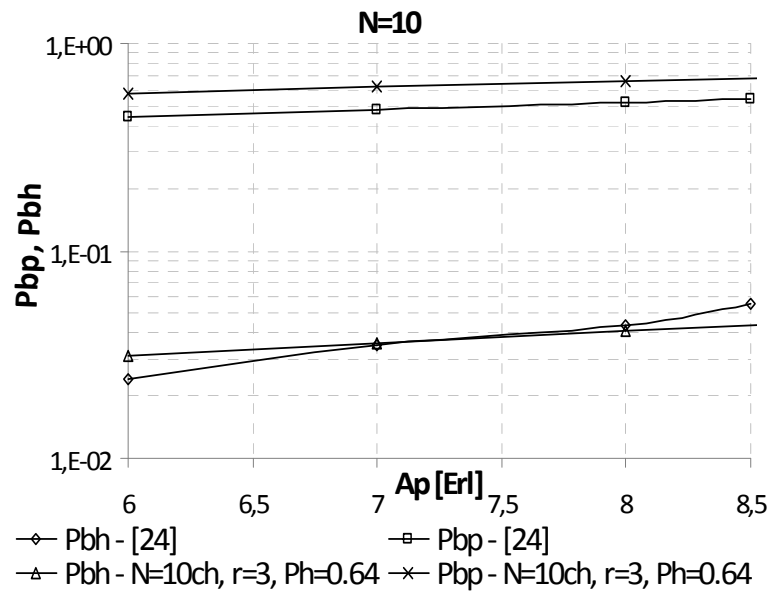

Fig. 13. The probability of primary calls blocking $\left(P_{\mathrm{bp}}\right)$ and handover calls dropping $\left(P_{\mathrm{bh}}\right)$ as a function of offered traffic $\left(A_{\mathrm{p}}\right)$.

\begin{tabular}{|c|c|c|}
\hline $\boldsymbol{R}[\mathbf{k m}]$ & $\boldsymbol{V}[\mathbf{k m} / \mathbf{h}]$ & $\boldsymbol{P}_{\mathbf{h}}$ \\
\hline 10 & 50 & 0.14 \\
\hline 10 & 80 & 0.2 \\
\hline 10 & 120 & 0.28 \\
\hline 1 & 5 & 0.14 \\
\hline 1 & 30 & 0.49 \\
\hline 1 & 50 & 0.61 \\
\hline
\end{tabular}

Tab. 2. The values of $P_{\mathrm{h}}$ for various $R$ and $V$.

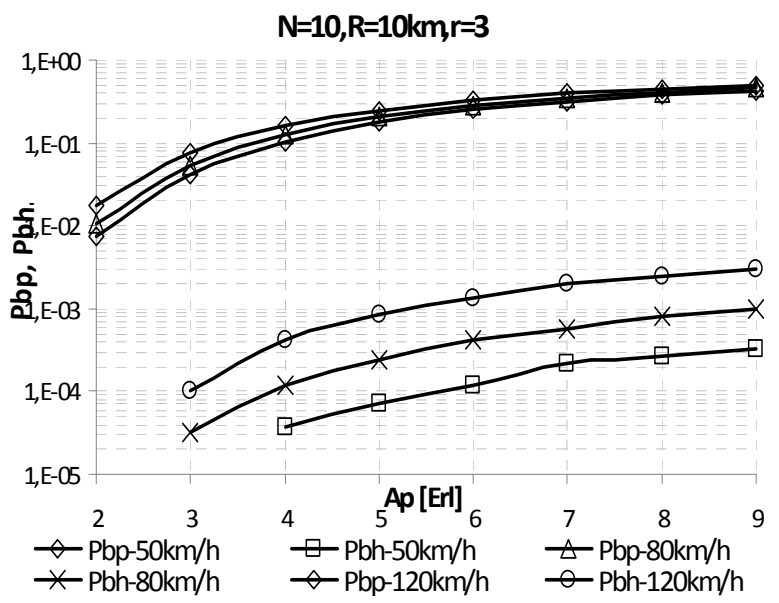

Fig. 14. The probability of primary calls blocking $\left(P_{\mathrm{bp}}\right)$ and handover calls dropping $\left(P_{\mathrm{bh}}\right)$ as a function of offered traffic $\left(A_{\mathrm{p}}\right)$ for various users' speed in rural areas.

Figure 14 presents probability of primary calls blocking $\left(P_{\mathrm{bp}}\right)$ and handover calls dropping $\left(P_{\mathrm{bh}}\right)$ as a function of offered traffic $\left(A_{\mathrm{p}}\right)$ in a typical rural cell with radius $R=10 \mathrm{~km}$. The number of reserved channels only for handover is $r=3$. The results are presented for user moving speed $V=50 \mathrm{~km} / \mathrm{h}, V=80 \mathrm{~km} / \mathrm{h}$ and $V=120 \mathrm{~km} / \mathrm{h}$. The increase of $V$ causes increase both in $P_{\mathrm{bp}}$ and $P_{\mathrm{bh}}$. This increase is more obvious when considering $P_{\mathrm{bh}}$. When $V$ increases from $50 \mathrm{~km} / \mathrm{h}$ to $120 \mathrm{~km} / \mathrm{h}, P_{\text {bh }}$ increases about ten times, while $P_{\mathrm{bp}}$ increases only about two times.

Figure 15 presents probability of primary calls blocking $\left(P_{\mathrm{bp}}\right)$ and handover calls dropping $\left(P_{\mathrm{bh}}\right)$ as a function of offered traffic $\left(A_{\mathrm{p}}\right)$ in a typical urban cell with radius 


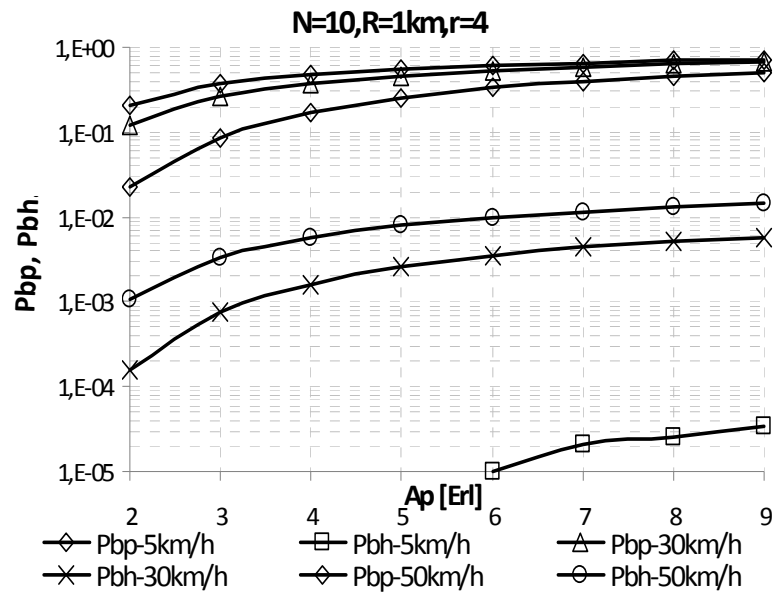

Fig. 15. The probability of primary calls blocking $\left(P_{\mathrm{bp}}\right)$ and handover calls dropping $\left(P_{\mathrm{bh}}\right)$ as a function of offered traffic $\left(A_{\mathrm{p}}\right)$ for various users' speed in urban areas.

$R=1 \mathrm{~km}$. The number of reserved channels only for handover is increased comparing to urban area case to $r=4$. The results are presented for user moving speed $V=5 \mathrm{~km} / \mathrm{h}$ (pedestrian speed), $V=30 \mathrm{~km} / \mathrm{h}$ and $V=50 \mathrm{~km} / \mathrm{h}$. The values of $P_{\mathrm{bp}}$ and $P_{\mathrm{bh}}$ are more sensitive to $V$ increase in the case of urban cell, because the cell radius is significantly lower $(R=1 \mathrm{~km})$ and $R$ and $V$ cause changes in $P_{\mathrm{bp}}$ and $P_{\mathrm{bh}}$ values by their mutual quotient.

The implementation of two-dimensional systems also allows us to give an answer on the following question: is the probability of handover call appearance equal in all sub-states $\{i, j\}$ where it is $i+j=N-r$ ? In other words, is it reasonable to reserve $r$ channels only for handover calls even if all channels are already seized by handover calls?

Let us suppose that $n(i, N-r-i)$ is the number of new handover calls, which are generated in the sub-state $\{i, j\}$ in the simulation process when it is $i+j=N-r$. The part of handover calls relative frequency which appear in this sub-state may be expressed by

$$
R F(i, N-r-i)=\frac{n(i, N-r-i)}{\sum_{i=0}^{N-r} n(i, N-r-i)} .
$$

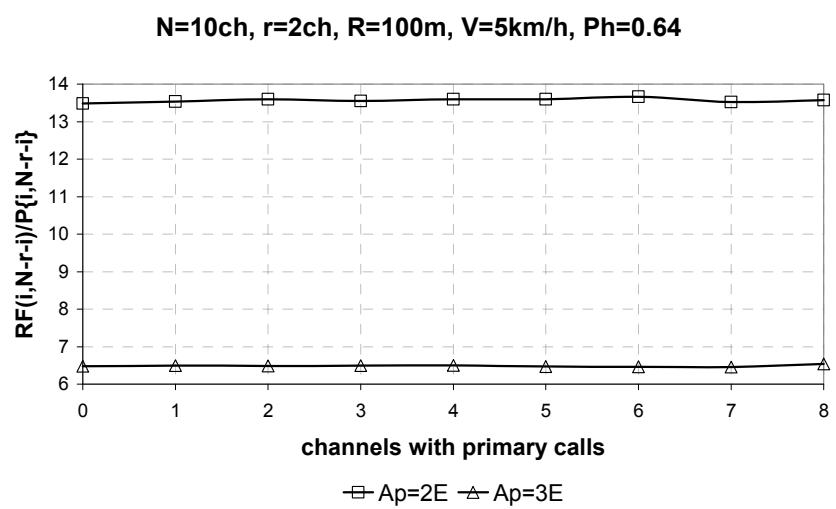

Fig. 16. The ratio of handover calls generation probability in the state $\{i, N-r-i\}$ to the probability of the same state as a function of the number of primary calls.
The number of new calls generated in some sub-state $\{i, j\}$ depends also on the probability that system is in this substate. That's why the variable $R F(i, N-r-i) / P\{i, N-r-i\}$ presents the variation of the new handover calls from one sub-state to the other. Figure 16 presents the variation of the variable $R F(i, N-r-i) / P\{i, N-r-i\}$ as the result of simulation for the model with $N=10$ channels when there are $r=2$ channels reserved only for handover. The results are presented for two offered traffic values $A_{\mathrm{p}}=2 \mathrm{E}$ and $A_{\mathrm{p}}=3 \mathrm{E}$. The variable $R F(i, N-r-i) / P\{i, N-r-i\}$ has approximately constant value for one value of offered traffic, which proves that the probability of new handover calls is the same in all sub-states.

\section{The Necessary Number of Iterations in the Analysis of System with Handover Calls}

Table 3 presents the necessary number of iterations when simulating the systems with implemented handover to obtain value of $P_{\mathrm{bp}}$, which does not differ more than $1.5 \%$ in two consecutive iterations.

Generally speaking, the necessary number of iterations for the cells with the small value of $P_{\mathrm{h}}$ is not too great, i.e. it may be expected that the results relatively quickly converge to their exact values.

The situation is different when $P_{\mathrm{h}}$ is higher. In this case the necessary number of iterations is significantly increased when the offered traffic is increased, thus extending the time needed for simulation (for $A_{\mathrm{p}}=5 \mathrm{E}$ it was necessary to have even 68 iterations).

It is possible to make a correction in the method for determination of new values of $P_{\mathrm{bp}}$ and $P_{\mathrm{bh}}$ for the next iteration step. The modification of the simulation program is presented in Fig. 17. This modification replaces the last block from the flow-chart already presented in Fig. 6. Instead of the output value of the probabilities $P_{\mathrm{bp}}$ and $P_{\mathrm{bh}}$ in an iteration to represent the input value for the next iteration, we introduced the following modification in the simulation process. For the each $10^{\text {th }}$ iteration the input values of $P_{\mathrm{bp}}$ and $P_{\mathrm{bh}}$ are calculated as the average value of these parameters at the output of last two iterations. In the case of

\begin{tabular}{|c|c|c|c|}
\hline \multicolumn{2}{|c|}{$N=22, r=3, P_{\mathrm{h}}=0.17$} & \multicolumn{2}{|c|}{$N=10, r=4, P_{\mathrm{h}}=0.64$} \\
\hline$A_{\mathrm{p}}(\mathrm{E})$ & $\begin{array}{l}\text { The number } \\
\text { of iterations }\end{array}$ & $A_{\mathrm{p}}(\mathbf{E})$ & $\begin{array}{l}\text { The number } \\
\text { of iterations }\end{array}$ \\
\hline 10 & 3 & 2 & 8 \\
\hline 11 & 3 & 3 & 15 \\
\hline 12 & 3 & 4 & 28 \\
\hline 13 & 3 & 5 & 68 \\
\hline 14 & 3 & 6 & \multirow{4}{*}{$>200$} \\
\hline 15 & 4 & 7 & \\
\hline 16 & 4 & 8 & \\
\hline $17-22$ & 4 & 9 & \\
\hline
\end{tabular}

Tab. 3. The necessary number of iterations when simulating the system with handover. 


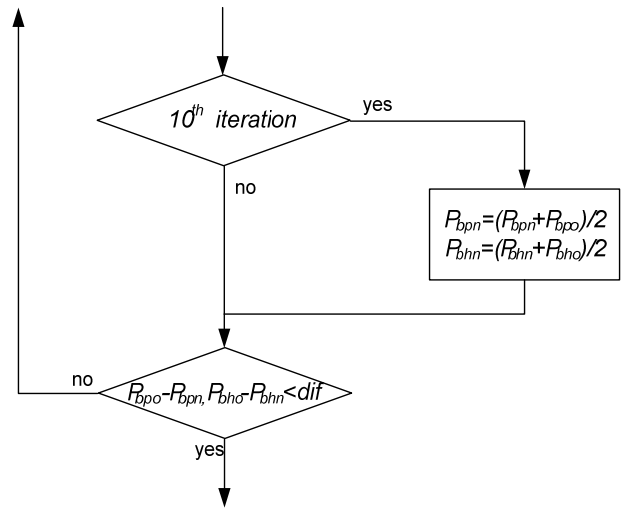

Fig. 17. The introduced modification to speed-up simulation results convergence.

implementation of such an algorithm the values $P_{\mathrm{bp}}$ and $P_{\mathrm{bh}}$ converge to their exact values significantly faster for $P_{\mathrm{h}}=0.64$ than it was before the modification is implemented. The necessary number of iterations after this modification is presented in Tab. 4. In the characteristic cases when the satisfactory result is obtained after 11 iterations (with 25 million generated random numbers in each iteration, i.e. each iteration has 25 million passes through program loop), the time necessary to perform a simulation is less than 1.5 minutes.

The number of necessary iterations in all cases presented by Tab. 4 is equal to 10 or a bit higher. During the simulation, the values of $P_{\mathrm{bp}}$ and $P_{\mathrm{bh}}$ in two consecutive iterations when considering first 9 iterations have a significant oscillatory behavior. The mean values of $P_{\mathrm{bp}}$ and $P_{\mathrm{bh}}$ between these two consecutive iterations are in the vicinity of the final $P_{\mathrm{bp}}$ and $P_{\mathrm{bh}}$ values. That's why the $10^{\text {th }}$ iteration settles down the oscillations. Namely, the values of $P_{\mathrm{bp}}$ and $P_{\text {bh }}$ after the $10^{\text {th }}$ iteration are calculated as the mean value of $9^{\text {th }}$ and first determined $10^{\text {th }}$ iteration.

Our limit of relative oscillations is fixed to $1.5 \%$, as already stated, and in our simulation trials oscillations after the $10^{\text {th }}$ iteration were very near to this value. In some cases (for example for $A_{\mathrm{p}}=3 \mathrm{E}$ and $A_{\mathrm{p}}=5 \mathrm{E}$ ) oscillations were lower than $1.5 \%$ and it was enough to have 11 iterations. For $A_{\mathrm{p}}=4 \mathrm{E}$ oscillations were a bit higher than $1.5 \%$ and it was necessary to have even 3 more iterations in the area of slow oscillations decrease (13 iterations total) to settle these oscillations below $1.5 \%$.

\section{Conclusions}

This paper presents a new, two-dimensional model for the analysis of networks with mobile users who are moving. In such a case the traffic properties of cells in network may be estimated more detailed when considering primary and handover traffic instead of the total traffic. The model is intended to determine performances of systems with the ability to decrease handover calls dropping probability by the reservation of a number of traffic channels only for handover calls. Two-dimensional model

\begin{tabular}{|c|c|}
\hline \multicolumn{2}{|c|}{$N=\mathbf{1 0}, \boldsymbol{r}=\mathbf{5}, \boldsymbol{P}_{\mathbf{h}}=\mathbf{0 . 6 4}$} \\
\hline $\boldsymbol{A}_{\mathbf{p}}(\mathrm{E})$ & The number of iterations \\
\hline 2 & 10 \\
\hline 3 & 11 \\
\hline 4 & 13 \\
\hline 5 & 11 \\
\hline 6 & 11 \\
\hline 7 & 11 \\
\hline 8 & 12 \\
\hline 9 & 12 \\
\hline
\end{tabular}

Tab. 4. The necessary number of iterations when simulating the system with handover after the modification of the algorithm for the determination of new $P_{\mathrm{bp}}$ and $P_{\mathrm{bh}}$ values.

allows us to determine some characteristics of the cells, which may not be determined by the implementation of existing one-dimensional models. All necessary cell characteristics are determined on the basis of sub-state probabilities. Three characteristics, which may not be determined from the one-dimensional model, are emphasized in this paper as an example: the probability that the same number of channels are seized by primary and by handover calls, the probability that there are no primary or no handover calls in the system and the probability of new handover calls generation as a function of instantaneous primary (handover) calls. The implementation of two-dimensional models to improve system performances by dynamic variations of the number of reserved channels as a function of relative relation between primary and handover traffic will be analyzed in the further development.

Reservation of some traffic channels only for handover calls very quickly leads to the significant decrease of the handover calls dropping probability in relation to primary calls. This behavior is illustrated in the paper by two examples, which prove that this effect is more significant in the cells with the greater number of traffic channels. It is analyzed how primary and handover call loss depend on the users' moving speed $V$ and the cell radius $R$. Two additional examples are intended to compare the performances of system with channel reservation to the system which has waiting queues for handover calls.

Simulation method, which is presented in this paper, is very important for the cells with the greater number of traffic channels when the calculation is very complex or even impossible. The specificity of calculation and simulation method for the traffic loss determination in the systems with handover is that exact result may not be obtained after one calculation, i.e. simulation cycle. It is proved in this paper that the necessary number of steps in the calculation, i.e. number of simulation cycles, depends on the value of handover probability $P_{\mathrm{h}}$. The determined values of system traffic characteristics (first of all traffic loss of primary and handover calls) converge to their exact values more quickly when the values of $P_{\mathrm{h}}$ are lower. That's why original modification for the calculation of new $P_{\mathrm{bp}}$ and $P_{\mathrm{bh}}$ values for the next iteration cycle is implemented in the paper. This modification is based on $P_{\mathrm{bp}}$ and $P_{\mathrm{bh}}$ traffic loss values in the last two iteration cycles. 


\section{Acknowledgments}

This paper is realized in the framework of the projects TR32051 and TR32007, which are financed by the Ministry of Education, Science and Technological Development of the Republic of Serbia.

\section{References}

[1] LEBL, A., MITIĆ, D., TRENKIĆ, B., et al. Determination of base station emission power change in a mobile network cell with movable users. Radioengineering, 2018, vol. 27, no. 4, p. 1174-1182. DOI: $10.13164 /$ re.2018.1174

[2] IVERSEN, V. B. Teletraffic Engineering and Network Planning. Denmark: Technical University of Denmark, 2015.

[3] POLLINI, G. P. Trends in handover design. IEEE Communications Magazine, 1996, vol. 34, no. 3, p. 82-90. DOI: $10.1109 / 35.486807$

[4] JABBARI, B. Teletraffic aspects of evolving and next-generation wireless communication networks. IEEE Personal Communications, 1996, vol. 3, no. 6, p. 4-9. DOI: $10.1109 / 98.556473$

[5] SCHWARTZ, M. Mobile Wireless Communications. Cambridge University Press, 2005. ISBN: 978-0521843478

[6] Agustina, J. V., ZHANG, P., KANTOLA, R. Performance evaluation of GSM handover traffic in a GPRS/GSM network. In Proceedings of the Eighth International Symposium on Computers and Communications (ISCC 2003). Kemer-Antalya (Turkey), 2003, p. 137-142. DOI: 10.1109/ISCC.2003.1214113

[7] ADEWALE, A. A., JOHN, S. N., ADAGUNODO, E. R. Performance comparison of dynamic guard channel assignment with buffered prioritized scheme for mobile WiMAX network. In Proceedings of the World Congress on Engineering 2016. London (UK), 2016, vol. I, p. 1-5. ISBN 978-988-19253-0-5, ISSN: 20780958

[8] BASHARIN, G. P., MERKULOV, V. E. Blocking probability analysis of new and handover calls in cellular mobile networks with repeated attempts. In Proceedings of the $7^{\text {th }}$ International Conference on Telecommunications (ConTEL 2003). Zagreb (Croatia), 2003, p. 273-278. DOI: 10.1109/CONTEL.2003.176920

[9] XHAFA, A. E., TONGUZ, O. K. Dynamic priority queueing of handover calls in wireless networks: An analytical framework. IEEE Journal on Selected Areas in Communications, 2004, vol. 22, no. 5, p. 904-916. DOI: 10.1109/JSAC.2004.826927

[10] BERALDI, R., MARANO, S., PALUMBO, E. Analysis of new priority queueing strategies for handoff and originating calls in mobile cellular radio systems. In IEEE Wireless Communications System Symposium. Long Island (NY, USA), 1995, p. 63-69. DOI: 10.1109/WCSS.1995.588484

[11] MARTINEZ-BAUSET, J., PLA, V., GARCIA-ROGER, D., et al. Admission control policies to minimize blocking/forcedtermination in multimedia cellular networks. Chapter 13 in: Ming, G., Pan, Y., Fan, P. (eds.) Advances in Wireless Networks: Performance Modelling, Analysis and Enhancement. Nova Science Publishers, Inc., 2008. ISBN: 1-60021-713-3

[12] KAWSER, M. T., ISLAM, M. R., RAHIM, M. R., et al. Versatile controllability over cell switching for speedy users in LTE HetNets. Radioengineering, 2017, vol. 26, no. 1, p. 179-190. DOI: $10.13164 /$ re 2017.0179
[13] KIRSAL, Y. Analytical modelling of a new handover algorithm for improve allocation of resources in highly mobile environments. International Journal of Computers Communications \& Control, 2016, vol. 11, no. 6, p. 789-803. DOI: 10.15837/ijccc.2016.6.2564

[14] THUMTHAWATWORN, T., PERVEZ, A., SANTIPRABHOB, P. Enhanced adaptive traffic dependent handover decision system for wireless mobile networks. International Journal on Advances in Networks and Services, 2014, vol. 7, no 1-2, p. 118-129. ISSN: 1942-2644

[15] JOVANOVIĆ, P., ŠUH, T., LEBL, A., et al. Influence of intra-cell connections on the traffic calculation of radio resources in mobile network. Frequenz, 2013, vol. 67, no. 9-10, p. 315-320. DOI: 10.1515/freq-2012-0124

[16] TOLSTRUP, M. Indoor Radio Planning - A Practical Guide for GSM, DCS, UMTS and HSPA. John Wiley and Sons, 2008. DOI: $10.1002 / 9781119973225$

[17] MARKOV, Ž. Calculation of the group of trunks containing faulty trunks by the theory of mixed telephone traffic. Archiv für Elektronik und Übertragungstechnik (AË̈), October 1988, vol. 42 , no. 5 , p. $285-287$.

[18] BENEŠ, V. E. Mathematical Theory of Connecting Networks and Telephone Traffic. Vol. 17, ch. 3: Rearrangeable networks, p. 82-135. New York: Academic Press, 1965. ISBN: 9780120875504

[19] ŠUH, T., MITIĆ, D., LEBL-ANTONIĆ, D., et al. Determination of the necessary number of technicians on the faculty. Acta Polytechnica Hungarica, 2014, vol. 11, no. 1, p. 21-36. DOI: 10.12700/aph.11.01.2014.01.2

[20] KOSTEN, K. M. Simulation in traffic theory. In Proceedings of the Sixth International Teletraffic Congress ITC. Munich (Germany), 1970, p. 411/1-411/8.

[21] MILEUSNIĆ, M., ŠUH, T., LEBL, A., et al. Use of computer simulation in estimation of GSM base station output power. Acta Polytechnica Hungarica, 2014, vol. 11, no. 6, p. 129-142. DOI: 10.12700/aph.11.06.2014.06.8

[22] LEBL, A., MITIĆ, D., PERIŠIĆ, Đ., et al. A simulation model of assistants' and technicians' engagement in processing generated requests at a university. Ingeniería e investigación, 2016, vol. 36 , no. 2, p. 35-42. DOI: $10.15446 /$ ing.investig.v36n2.54210

[23] MITIĆ, D., LEBL, A., MILEUSNIĆ, M., et al. Traffic simulation of GSM cells with half-rate connection realization possibility. Journal of Electrical Engineering, 2016, vol. 67, no. 2, p. 95-102. DOI: $10.1515 /$ jee-2016-0014

[24] MITIĆ, D., MARKOV, Ž., KOSJER, V., LEBL, A. Programs for mobile systems simulation, [Online] Available at: http://www.iritel.com/index.php/en/design-services/646-pprgramsfor-mobile-systems-simulation

[25] GARAH, M., BENATIA, D. Handover prioritizing scheme for reducing call failure probability in cellular wireless network. Wireless Communications and Mobile Computing, 2009, vol. 9, p. $1660-1667$, DOI: $10.1002 / \mathrm{wcm} .755$

\section{About the Authors ...}

Dragan MITIĆ was born in Belgrade, Serbia, in 1953. He received his B.Sc. and M.Sc. from the Faculty of Electrical Engineering in Belgrade, Republic of Serbia, in 1977, and 1984, respectively, and his Ph.D. from the Faculty of Technical Science in Novi Sad, in 2002. Dr. Mitić is a science advisor in IRITEL, Institute for Electronics and 
Telecommunications, Belgrade, Serbia. From 1977 until 1989 he was employed at the Land Forces Military Technical Institute in Belgrade, and since 1989 in IRITEL. He is author or co-author of more than 160 international and national scientific and professional papers. He works on several research projects for equipment of specific applications.

Aleksandar LEBL was born in Zemun, Serbia, in 1957. He received his B.Sc. and M.Sc. from the Faculty of Electrical Engineering in Belgrade, Republic of Serbia, in 1981 and 1986, respectively, and his Ph.D. from the Faculty of Technical Science in Novi Sad, in 2009. He was employed from 1981 in the Switching Department of the Institute for Electronics and Telecommunications IRITEL in Belgrade and from 2015 he is in Radio Communication Department, also in IRITEL. During years he worked on the project of Digital Switching System for Serbian Telecommunication Industry.

Žarko MARKOV was born in Žitište, Serbia, in 1946. He received his B.Sc., M.Sc. and Ph.D. from the Faculty of Electrical Engineering in Belgrade, Republic of Serbia, in
1969, 1975 and 1976, respectively. Dr. Markov is a scientific counsellor in IRITEL, Institute for Electronics and Telecommunications, Belgrade, Serbia. Area of work: Switching technics, teletraffic theory, network signaling. $\mathrm{He}$ is author or co-author of more than hundred papers and six books. At the University of Belgrade, School of Electrical Engineering, Dr. Markov was a professor at the course of Switching Technics and Network Signaling.

Vladimir KOSJER was born in Inđija, Serbia, in 1989. He received his B.Sc. and M.Sc. from the Faculty of Technical Sciences in Novi Sad, Republic of Serbia, in 2012 and 2016, respectively, and started Ph.D. studies in 2019 at the same Faculty. During 2014/15, he was employed in EBV as a Field Application Engineer, and from 2015-2019 in RT-RK National Research Institute as software/firmware developer on different projects, e.g. 3D surround sound, Android based smart STB devices and in automotive segment, driving assistance. During the period of 2 school years, he was employed as teaching Assistant at the Faculty of Technical Sciences. He is currently employed in IRITEL in Radio Communication Department. 